\title{
Local Field Potentials and Spikes in the Human Medial Temporal Lobe are Selective to Image Category
}

\author{
Alexander Kraskov ${ }^{1}$, Rodrigo Quian Quiroga ${ }^{1,2}$, Leila Reddy ${ }^{3}$, \\ Itzhak Fried $^{4,5}$, and Christof Koch $^{1}$
}

\begin{abstract}
Local field potentials (LFPs) reflect the averaged dendrosomatic activity of synaptic signals of large neuronal populations. In this study, we investigate the selectivity of LFPs and single neuron activity to semantic categories of visual stimuli in the medial temporal lobe of nine neurosurgical patients implanted with intracranial depth electrodes for clinical reasons. Strong selectivity to the category of presented images was found for the amplitude of LFPs in $8 \%$ of implanted microelectrodes and for the firing rates of single and multiunits in $14 \%$ of microelectrodes. There was little overlap between the LFP- and spike-selective microelectrodes. Separate analy-
\end{abstract}

\section{INTRODUCTION}

Existing experimental approaches to investigate the functions of the brain by recording electrical signals vary from single-cell recordings in animals to surface electroencephalography (EEG) in humans. Animal electrophysiology usually relies on the analysis of spiking activity of neurons, while placing less emphasis on local field potentials (LFPs), a slow nonspiking component of the recorded electrical signal. In contrast, studies of brain electrical activity in humans typically use electrical potentials recorded from the surface of the skull. Such EEG signals represent the electrical activity of large fraction of cortical and subcortical tissue but can still be linked to different behavioral states or cognitive functions.

Certain neurological conditions, in particular, pharmacologically intractable epilepsy, require, on occasion, the implantation of either subdural electrodes that rest on the surface of the cortex, or of depth electrodes that are implanted into the brain parenchyma (Engel, Moll, Fried, \& Ojemann, 2005; Lachaux, Rudrauf, \& Kahane,

\footnotetext{
${ }^{1}$ California Institute of Technology, ${ }^{2}$ University of Leicester, UK, ${ }^{3}$ Massachusetts Institute of Technology, ${ }^{4}$ University of California at Los Angeles, ${ }^{5}$ Tel-Aviv University, Israel
}

sis of the power and phase of LFPs revealed that the mean phase was category-selective around the $\theta$ frequency range and that the power of the LFPs was category-selective for high frequencies around the $\gamma$ rhythm. Of the 36 microelectrodes with amplitude-selective LFPs, 30 were found in the hippocampus. Finally, it was possible to readout information about the category of stimuli presented to the patients with both spikes and LFPs. Combining spiking and LFP activity enhanced the decoding accuracy in comparison with the accuracy obtained with each signal alone, especially for short time intervals.
2003; Bechtereva \& Abdullaev, 2000). The signals obtained with these intracranial electrodes represent average activity of the brain with temporal and spatial resolution on the order of milliseconds and centimeters, respectively. The size and impedance of these clinically used electrodes typically do not permit the recording of spiking activity of neurons. Here we present data obtained with microelectrodes implanted in the human medial temporal lobe (MTL) of epilepsy patients. Their impedance $(\sim 0.5 \mathrm{M} \Omega)$ and size (diameter of the tip $\sim 40 \mu \mathrm{m}$ ) enabled us to record spiking activity of single neurons as well as LFPs (Fried et al., 1999). The relationship between LFPs and spiking activity in nonprimates was already addressed as early as the 1960s (see Haberly \& Shepherd, 1973; John, 1967, 1972; Fromm, 1967; Buchwald, 1965 and Logothetis, 2003, for review). There were also several studies dealing with the correlation of spiking and epileptiform activity in epileptic patients (Wyler, Ojemann, \& Ward, 1982; Verzeano, Crandall, \& Dymond, 1971). In monkey electrophysiology, LFPs and their relationship to spiking activity have been actively studied only in the last few years (Kreiman et al., 2006; Liu \& Newsome, 2006; Henrie \& Shapley, 2005; Scherberger, Jarvis, \& Andersen, 2005; Mehring et al., 2003; Pesaran, Pezaris, Sahani, Mitra, \& Andersen, 2002). For example, the LFP activity in the parietal cortex 
of macaque monkeys was found to discriminate between preferred and antipreferred directions of reach plans (Pesaran et al., 2002). The LFPs in monkey motor cortex were also predictive of hand movements (Mehring et al., 2003). Scherberger et al. (2005) reported that the monkey behavioral state can be decoded with LFPs better than with the spiking activity. Increasing stimulus contrast was found to cause an increase in power, in particular, in gamma band, of the LFP recorded in macaque V1 (Henrie \& Shapley, 2005). Moreover, it occurred over a contrast range within which the spike rates of cortical cells were saturating. Recently, LFPs, as well as spiking activity recorded in the inferior temporal cortex (IT) of macaque monkeys, were shown to be object-selective but barely related to each other (Kreiman et al., 2006). The aim of our study is to investigate in humans, the selectivity of spiking and LFP activity recorded simultaneously from the same microelectrodes during a simple perceptual task, and the relationship between their selectivities.

The selectivity of spiking activity recorded in the human MTL to visual categories has already been reported by our group (Kreiman, Koch, \& Fried, 2000). In this study, we investigated visual category selectivity of the spiking activity and LFPs in a different group of patients using the amplitude, power, and mean phase of the LFPs. In addition, we compared selectivity properties of the LFP and spiking activity and found a weak correlation between them. We were also able to decode information about the category of a presented stimulus using the spiking and LFP activity separately and together. Decoding accuracy in a short time window was found to be optimal using the LFPs and spiking activity simultaneously as an input to the decoding algorithm. The weak correlation of object selectivity properties of the LFPs and spiking activity and augmented decoding accuracy using both of them support the hypothesis that the LFPs contain additional information about the category of a visual stimulus.

\section{METHODS}

\section{Subjects and Recordings}

The data came from 12 sessions in nine patients with pharmacologically intractable epilepsy (all right-handed, 4 men, 17 to 47 years old). This set of patients overlaps with the one used in a previous study (Quian Quiroga, Reddy, Kreiman, Koch, \& Fried, 2005), but the current data corresponds to different experimental sessions. For these patients, extensive noninvasive monitoring did not yield concordant data corresponding to a single resectable epileptogenic focus. Therefore, they were implanted with chronic depth electrodes for 7-10 days to determine the seizure focus for possible surgical resection (Fried, MacDonald, \& Wilson, 1997). Here we report data from microelectrodes in the hippocam- pus, amygdala, entorhinal cortex, and parahippocampal gyrus. All studies conformed to the guidelines of the Medical Institutional Review Board at UCLA. The electrode locations were based exclusively on clinical criteria and were verified by magnetic resonance imaging (MRI) or by computed tomography (CT) coregistered to preoperative MRI. Each electrode probe had a total of nine microwires at its end, eight active recording channels and one reference. The differential signal from the microwires was amplified using a 64-channel Neuralynx system (Tucson, AZ), filtered between 1 and $9000 \mathrm{~Hz}$ and sampled at $28 \mathrm{kHz}$. Spike detection and clustering was done using recordings band-pass filtered between 300 and $3000 \mathrm{~Hz}$. The LFPs were obtained by band-pass filtering the same recordings between 1 and $100 \mathrm{~Hz}$ and down-sampling them to $256 \mathrm{~Hz}$.

Each recording session lasted about $30 \mathrm{~min}$. Subjects were sitting in bed, facing a laptop computer, on which pictures of individuals, animals, landmarks, or objects were shown. The images covered about $1.5^{\circ}$, were centered on a laptop screen, and were displayed six times each in pseudorandom order for 1 sec. Images were photos of animals, landmarks, and celebrities, which were partially chosen according to the patients' preferences, as well as photos of people and places unknown to the patients. More details about the stimulus set are available in Quian Quiroga et al. (2005). The interstimulus interval (ISI) was randomized with the minimum ISI equal to $1.5 \mathrm{sec}$. In order to encourage subjects to attend to the picture presentations, they had to respond whether the pictures contained a face or something else by pressing the "Y" and " $\mathrm{N}$ " keys, respectively.

\section{Data Analysis}

We analyzed the signals recorded from 568 microelectrodes implanted in different locations of the human MTL. For the LFP data, we initially applied a digital notch filter at $60 \mathrm{~Hz}$ and the first two harmonics (4th-order elliptic filter, $0.1 \mathrm{~dB}$ peak-to-peak ripple, $40 \mathrm{~dB}$ stopband attenuation). Recordings that showed either peaks at harmonics of $60 \mathrm{~Hz}$ on the power spectrum or highfrequency noise were discarded, thus obtaining a final set of 451 "clean" microelectrodes for LFP analysis, 384 of which showed spiking activity.

We discarded from the analysis trials having more than 5 points outside of the mean \pm 5 standard deviation range. The mean and the standard deviation were calculated across all trials for each sample point.

\section{Analysis of the LFP Amplitude Selectivity}

To quantify category selectivity of the LFP amplitude, we applied a sample-by-sample one-way analysis of variance (ANOVA) (Blair \& Karniski, 1993; Guthrie \& Buchwald, 1991) with the category identity as a main factor to the 
LFP values. The sample-by-sample ANOVA test gives a time-resolved significance level of how the LFP values are different across categories. We considered a microelectrode to be selective to a category if at least 15 consecutive points $(\sim 60 \mathrm{msec})$ of the ANOVA trace crossed the significance threshold of .001. To check whether the ANOVA traces crossed the .001 threshold by chance, we applied two control tests. First, we applied the ANOVA test to the one-second interval preceding stimulus onset. Secondly, we applied a bootstrap procedure by shuffling the pictures in-between categories. Such shuffling destroys information about the category but preserves time correlations of LFPs and correlations between different presentations of the same picture. Because the bootstrap procedure is time consuming, we applied it only to the selective microelectrodes (one-way ANOVA, $p<.001,15$ consecutive points). The goal of the bootstrap test was to confirm that the significant $p$ values obtained with the ANOVA test were reflecting differences in averaged LFPs for semantic categorization but not for other possible ones, for example, the cases when there was only one very strong response to a single picture.

\section{Latencies}

The latency of the selectivity for the averaged LFPs was defined as the time point when the LFPs for three categories started to be significantly different from each other, that is, when the ANOVA trace first crossed the significance level of $p=.001$ for at least 15 consecutive points. Analogously, for the definition of the latency of responsiveness, we applied a sample-by-sample $t$ test comparing the distribution of LFP values for each category and each time point during stimulus presentation, with the distribution of all LFP values during the baseline interval. The moment when the $t$ test trace first crossed significance level of $p=.001$ for at least 15 consecutive points for one category was defined as the latency of the responsiveness.

\section{Phase and Power Analysis}

To estimate the instantaneous phase and power, we used the continuous wavelet transform. The LFP of each trial was convoluted with complex Morlet wavelets $\Psi\left(f_{0}\right.$, $t)=\left(\sigma^{2} \pi\right)^{-1 / 4} \exp \left(-t^{2} / 2 \sigma^{2}\right) \exp \left(2 \pi i f_{0} t\right)$, where $f_{0}$ is the central frequency and $\sigma$ specifies the width of the wavelet in time domain. The Morlet wavelet is a complex sine wave whose amplitude is tapered by a Gaussian function. A wavelet family is characterized by a constant $n_{\mathrm{c}}=2 \pi f_{0} \sigma$, which we set equal to 6 . The convolution with a complex Morlet wavelet gives a series of complex wavelet coefficients $W\left(f_{0}, t\right)=\int \Psi\left(f_{0}, t-\tau\right) s(\tau) \mathrm{d} \tau=$ $A\left(f_{0}, t\right) \exp \left(i \phi\left(f_{0}, t\right)\right)$. From the wavelet coefficients corresponding to each frequency and time point, it is pos- sible to define the instantaneous power as $\left|W\left(f_{0}, t\right)\right|^{2}$, and the instantaneous phase as $\phi\left(f_{0}, t\right)$. To resemble the main EEG frequency bands, we used 11 frequencies spaced on the approximately logarithmical scale between 2.5 and $85 \mathrm{~Hz}$. Because the distribution of the power was found to be significantly different from Gaussian, we used a nonparametric sample-by-sample ANOVA (Kruskal-Wallis test) to test for selectivity of the LFP power. To test for mean-phase selectivity of the LFP, we used a sample-by-sample analysis of a common mean direction. This test is a generalization of a $t$ test analog for circular data to more than two variables.

Because, in general, the correlation between consecutive time points is higher for the lower frequencies, both for the power and phase analyses, we required that the significant difference between categories (with $p<.001$ ) lasted for at least two periods (Rizzuto et al., 2003). Due to the fact that a two-period interval may be relatively short, especially for high frequencies (e.g., for $85 \mathrm{~Hz}$ it is only about $23 \mathrm{msec}$, which corresponds to 6 points if the sampling frequency is $256 \mathrm{~Hz}$ ), the test for selectivity ( $p<.001$, two periods) was validated with a bootstrap procedure where the pictures were shuffled in-between the categories.

\section{Decoding}

A trial-by-trial decoding was done with a linear Fisher algorithm (Duda, Hart, \& Stork, 2001). We employed a one versus all strategy, that is, for each trial, a decision about its category was made based on the distributions of all other trials. The decoding accuracy was defined as the relative number of correct predictions. The chance level was equal to the inverse number of categories (.33). Time profiles of decoding accuracies were calculated using the number of spikes or mean LFP values in sliding time windows as an input to the decoding algorithm. The sliding windows had 50\% overlap. To increase the number of inputs to the classifier, we decreased the significance threshold to .01 in comparison to .001, which was used for selectivity analysis. We also calculated the decoding accuracies with a very loose significance threshold of .1, which did not change significantly the results.

\section{RESULTS}

We studied the spiking activity and LFPs recorded from the same microelectrodes implanted in the MTL of human subjects with pharmacologically intractable epilepsy. The placement of the electrodes was determined exclusively by clinical criteria (Fried et al., 1997). In 12 experimental sessions with nine patients, we recorded activity from 568 microelectrodes. Only "clean" recordings (451 microelectrodes) were used for further analysis (Methods). The microelectrodes were located in the 
amygdala (120), hippocampus (182), entorhinal cortex (102), and parahippocampal gyrus (47). Anatomical locations of microelectrodes were selectively estimated from the fused image of structural MRI taken before implantation of the electrodes and CT taken while the electrodes were implanted (Fried et al., 1999).

\section{Selectivity of the Amplitude of Local Field Potentials to Categories}

All images were divided into three semantic categories: faces, places, and animals. LFPs were time-aligned to the stimulus onset. Intervals of $1 \mathrm{sec}$ before and $1 \mathrm{sec}$ after stimulus onset were used in the analysis. Examples of averaged LFPs, raster plots, and poststimulus time histograms for three categories are shown in Figure 1. Here the spiking activity was clearly responsive and selective to the category "places," increasing from its background rate of about $0.2 \mathrm{~Hz}$ to approximately $2 \mathrm{~Hz}$. Yet surprisingly, the amplitude of the averaged LFPs for the category "places" was the smallest among the three categories. The averaged amplitudes of LFPs for the two other categories ("animals" and "faces") were significantly different from baseline. Another example is shown in Figure 2. Here the spiking activity, as well as LFPs, for the category "faces" was significantly different from the responses to images from the other two categories. The LFPs for the three categories from Figure 2 are plotted in Figure 3A. The corresponding ANOVA trace (Methods) is shown in Figure $3 \mathrm{~B}$. Note that after stimulus onset, there are two intervals corresponding to positive and negative reflections of the averaged LFPs from baseline where the ANOVA trace is far above the chosen significance value of .001. To verify the category selectivity of the LFPs, we applied two control tests (Methods). Note that for the example in Figure 3, there was no single point before stimulus onset where the amplitude of the LFPs was selective. In fact, none of the 451 microelectrodes showed selectivity during the baseline interval. In total, we found that 36 of the 451 microelectrodes $(8.0 \%)$ produced LFPs with a significant category selectivity (one way ANOVA, $p<.001,15$ consecutive points, bootstrap 1000 shuffles; Methods). Thirty of these microelectrodes were located in the hippocampus, three in the amygdala, three in the entorhinal cortex, and none in the parahippocampal gyrus (Figure 4). We did not find any significant difference $(p>.05$, binomial test) in the number of LFP- amplitude-selective microelectrodes between different hemispheres (left 19/251, right 17/200). An equal number of selective microelectrodes were found in the epileptogenic temporal lobe and in the contralateral lobe (18/270 versus 18/181).

The latency of the selectivity (i.e., the moment when the LFPs of the three categories started to be significantly
Figure 1. Spike and LFP responses from a microelectrode in the left medial hippocampus. For each of the three image categories shown, (A) raster plots, (B) poststimulus time histogram, and (C) average LFPs (thick) \pm 1 SEM across trials (thick, thin) are plotted. Vertical dashed lines indicate onset (zero on time axis) and offset $(1 \mathrm{sec})$ of the image. The number of pictures in a category is specified by the number in the brackets following the name of the category. Each picture was shown six times. The baseline firing rate was about $0.2 \mathrm{~Hz}$ for all three categories. Only during presentation of "place" pictures did the firing activity increase to $2 \mathrm{~Hz}$. In contrast, the average LFPs for "places" had the smallest amplitude, whereas the maximum amplitude of the average LFPs for the categories "animals" and "faces" was about $50 \mu \mathrm{V}$. The baseline amplitudes for all three categories were about $15 \mu \mathrm{V}$.

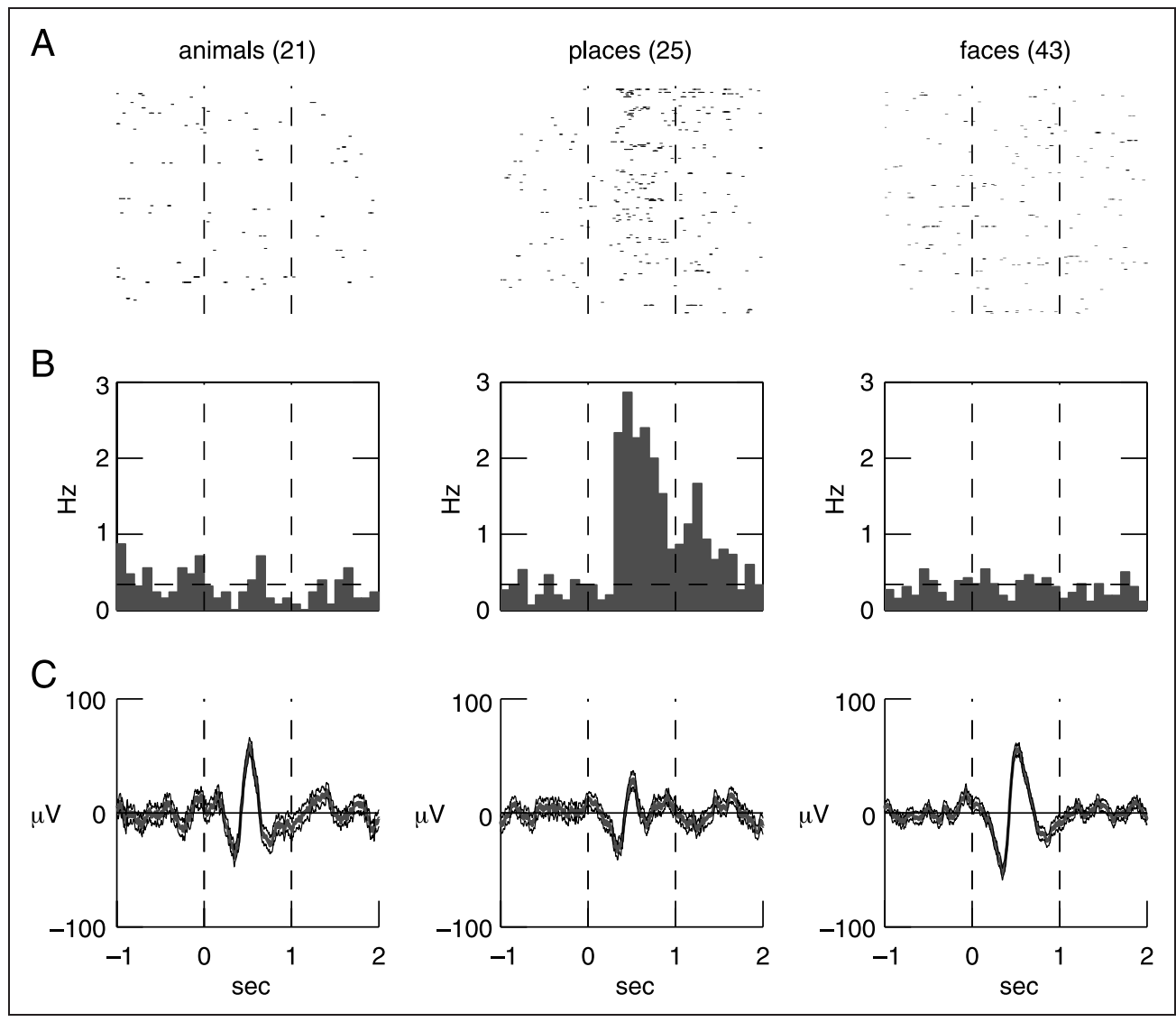


Figure 2. Spike and LFP responses from a microelectrode in the left anterior hippocampus. The baseline rate of this unit was $10.2 \mathrm{~Hz}$. It decreased significantly $(p<.001, t$ test $)$ upon presentation of animal pictures, remained unchanged for "places," and increased significantly $(p<.001, t$ test $)$ for "faces." The LFPs for "faces" were significantly different from baseline $(p<$ .001 , ANOVA, see Figure 3).

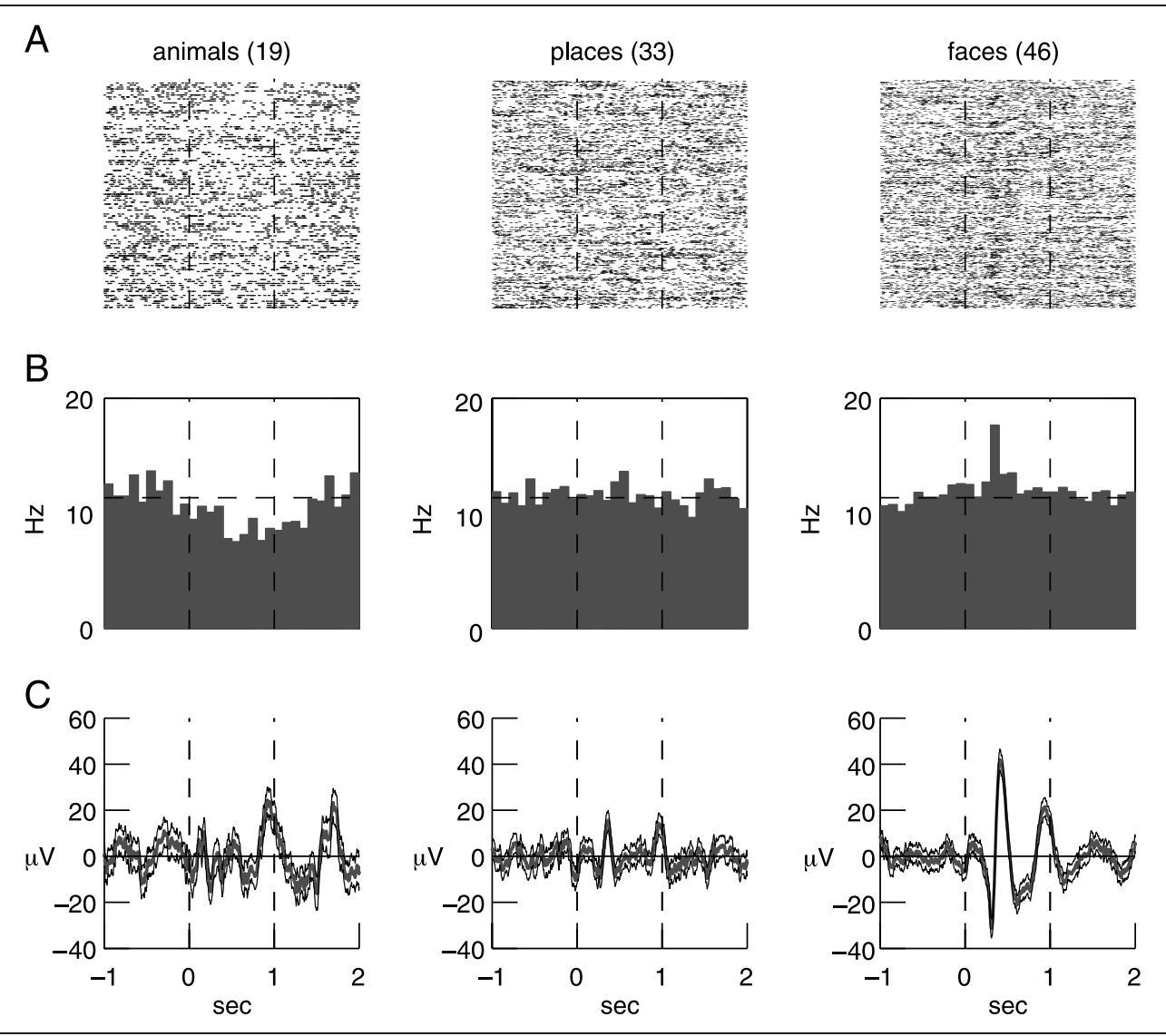

different from each other) was $460 \pm 15$ msec (mean \pm $S E M)$. For the selective microelectrodes, we also calculated the latency of the LFP responsiveness (i.e., when LFPs of a category started to be significantly different from the baseline [Methods] and found it to be $369 \pm 53 \mathrm{msec}$ $[$ mean $\pm S E M)$, which is significantly shorter than the latency of the selectivity ( $p<.01, t$ test). This difference is explained by the observation that, very often, the LFPs of
Figure 3. Time-resolved selectivity analysis. Selectivity of the LFP from the microelectrode of Figure 2. (A) Averaged LFPs (thick line) for the three categories \pm 1 SEM (thin lines). (B) Normalized $p$ values $\left(-\log _{10}(p)\right)$ obtained from the ANOVA test. The dash-dotted line corresponds to a significance of $p=.001$. The significance values are in logarithmic scale, significance less than .001 corresponds to the values on $y$-axis larger than $-\log _{10}(.001)=3$. The ANOVA test showed a significant difference among categories in two intervals of about 50 and 100 msec duration, corresponding to positive and negative reflections of the averaged LFPs for the category "faces."
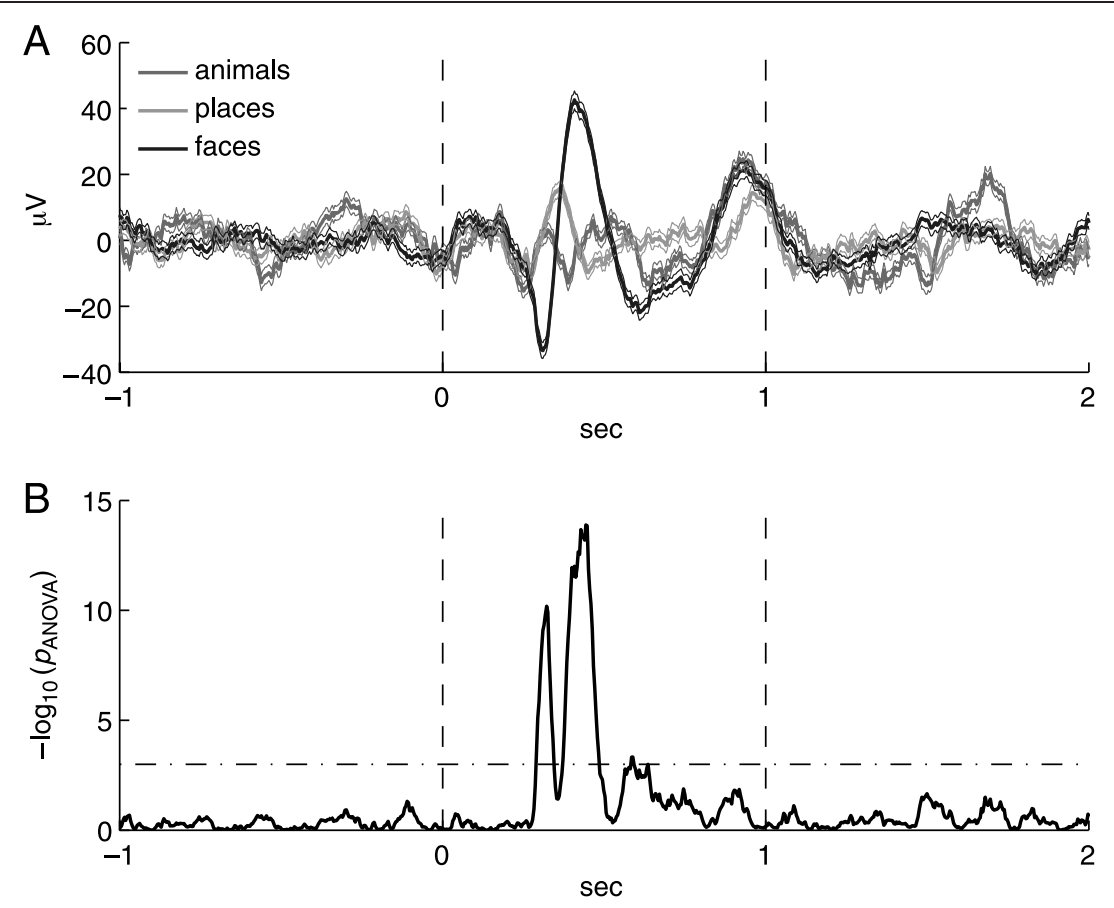
Figure 4. Localization of selective microelectrodes. (A) Total number of categoryselective microelectrodes across different brain regions [hippocampus (Hipp), amygdala (Am), parahippocampal gyrus (PG), entorhinal cortex (EC)]. (B) The number of selective microelectrodes normalized to the number of electrodes implanted in the area.

The vast majority of the amplitude-selective microelectrodes were found in the hippocampus. Spikeselective microelectrodes were more evenly distributed across these four regions.

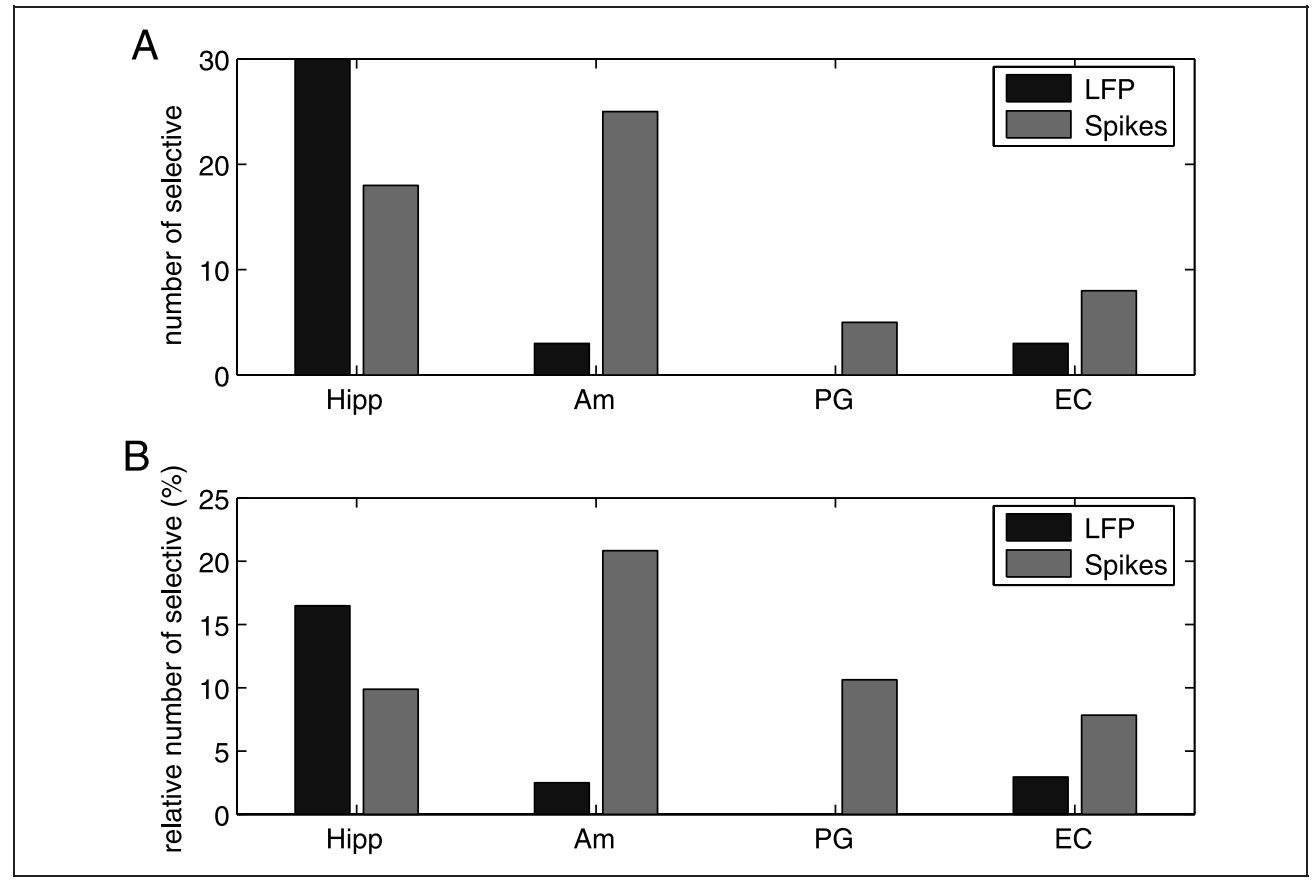

all three categories first start to deviate from the baseline and only later from each other (Figure S2).

\section{Selectivity of the Mean Phase and Power of the LFPs}

In addition to the selectivity of the LFP amplitudes, we studied whether their phase and power were categoryselective. The instantaneous phase and power of each category were defined for every time point in the interval [-1:2] sec using a complex Morlet wavelet transform (Grossmann, Kronland-Martinet, \& Morlet, 1989), widely used in EEG analysis (TallonBaudry, Bertrand, Delpuech, \& Pernier, 1997) (Methods). We applied a sample-by-sample, one-way, nonparametric ANOVA to the values of the power in different frequency bands. This gave a nonparametric ANOVA trace for each microelectrode and frequency band. Similarly, to quantify the category selectivity of the mean phases, we applied a sample-by-sample test for a common mean direction (Fisher, 1995) (Methods). In Figure 5, an example of a power-selective microelectrode is presented. The upper panel shows the averaged log-transformed power in the $\gamma$ band (the central frequency of the Morlet wavelet was $45 \mathrm{~Hz}$ ) for the three categories. Around $320 \mathrm{msec}$, the power of the category "faces" starts to be clearly different from the baseline power and from the power in the other two categories, the latter being reflected in the ANOVA trace plot (lower panel). An example of a mean phase-selective microelectrode is presented in Figure 6. The averaged LFPs filtered in $\theta$ band (the central frequency of the Morlet wavelet was $6 \mathrm{~Hz}$ ) for the three categories are shown in Figure 6A. Figure 6B shows the significance of the sample-by-sample test for a common mean direction. The test reached its maximum significance around 300 msec. The phase distributions and their mean direction at this particular time are shown in Figure $6 \mathrm{C}$. The length of the mean direction vector is proportional to the difference between the phase distribution for a given category from the uniform distribution (Rayleigh test). Figure 7A plots the total number of the microelectrodes showing selectivity to a category with the power and Figure $7 \mathrm{~B}$ with the mean phase across different frequency bands. The percentage of microelectrodes that showed selectivity with power $(24 ; 5.3 \%)$ or mean phase $(27 ; 6.0 \%)$ was relatively small. Comparing the data presented in Figure 7A and B, one can see a higher percentage of mean phase-selective LFPs for the lower frequencies and an opposite trend for the power-selective LFPs, namely, higher percentage of power-selective LFPs for higher frequencies (30-100 Hz). It leads to the intriguing hypothesis that there are two possible different mechanisms for the selectivity of LFPs. One involves phase locking in the lower frequencies and another one engages power increase in higher frequencies.

Half (13 microelectrodes) of the mean phase-selective microelectrodes were also selective for the amplitude of the LFPs (dark blue bars in Figure 7B), whereas only four microelectrodes showed selectivity for both power and the amplitude of the LFPs (dark blue bars in Figure 7A). This is not very surprising because the activity phase-locked to the stimulus onset is mostly preserved in the averaged LFPs, whereas the induced, nonstimulus-locked activity is averaged out and is revealed only in the averaged power. 
Figure 5. Example of a microelectrode with selective LFP power. (A) Averaged log-transformed power in the $\gamma$ band with a central frequency of the complex Morlet wavelet at $45 \mathrm{~Hz}$ (thick line) for three categories \pm 1 SEM (thin lines). (B) Normalized $p$ values obtained from ANOVA test. The dash-dotted line corresponds to the significance level $p=.001$. The averaged instantaneous power is larger for the category "faces" than the power for the other two image categories for about 100 msec starting at $\sim 300$ msec.

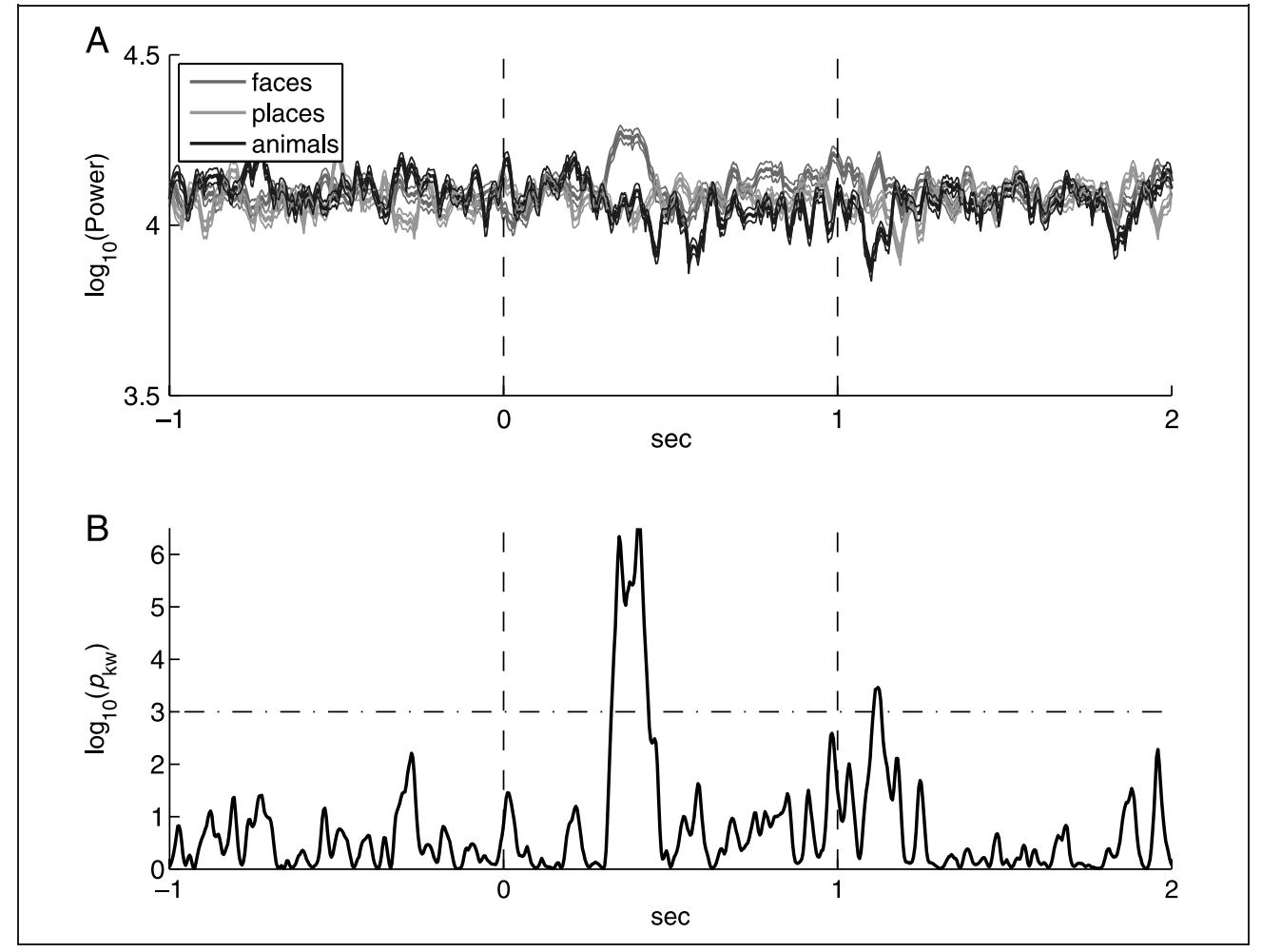

\section{Selectivity of Spiking Activity to Categories}

The spiking activity recorded with the same microelectrodes used to record the LFPs was preprocessed using a novel spike sorting algorithm (Quian Quiroga, Nadasdy, \& Ben-Shaul, 2004). To quantify the category selectivity of the spiking activity, we applied an one-way ANOVA with the category identity as a main factor, and the number of spikes in the interval [300:1000] msec (Quian Quiroga et al., 2005) as repeated measures. A $t$ test comparison with the baseline interval [-1000:-300] msec was used as post hoc test to define the responsive category. Additionally, we performed a bootstrap test. The pictures were randomly shuffled between categories and an ANOVA test was applied. We found that 66 out of the 591 recorded units (11.2\%) had a spiking response with significant category selectivity (ANOVA, $p<.001, t$ test, $p<.001$, bootstrap 1000). Fourteen units showed a significant decrease in firing rate, and eight units showed a significant increase to one category and a significant decrease in firing rate to another one.

Units selective to at least one of the three semantic categories were recorded from 56 of the 384 microelectrodes (14.6\%) used in the analysis. These numbers are comparable with those reported in Kreiman et al. (2000). As a control, the baseline and stimulus presentation intervals were exchanged and the same analysis was repeated. In this case, only one significant response was found. The distribution of the spike-selective microelectrodes across different brain regions was more uni- form in comparison to that of the LFPs. We found 18 selective microelectrodes in hippocampus, 25 in the amygdala, 8 in entorhinal cortex, and 5 in parahippocampal gyrus (Figure 4).

To compare the latency of the selective spiking and LFP activities, we convolved each spike train with a Gaussian kernel (100 msec width at half height) and repeated the same analysis used for the selectivity of the LFPs amplitudes (sample-by-sample one-way ANOVA, $p<.001,15$ consecutive points). The latency of spikeselectivity was defined as the first time point when there was a significant difference between the categories. The spike-responsiveness latency was found equivalent to the latency of selectivity. The average value for the latency of selectivity was found to be $341 \mathrm{msec}(14 \mathrm{msec} S E M)$. It was significantly ( $p<.001, t$ test) earlier then the latency of selectivity of the LFPs. Yet, the latency of the spiking responses was found to be not significantly different from the latency of the LFP responses ( $t>0.3, t$ test).

For the spiking activity, we found $24 / 315$ category selective units in the left hemisphere and 42/276 in the right hemisphere, and 20/343 category selective units in the epileptogenic hemisphere and 46/248 on the contralateral one. We found more category-selective units in the contralateral side. Because we do not have extensive patient statistics (9 patients), we cannot make any conclusive claims about lateralization of the category selectivity effect.

In total, we found 85 microelectrodes which produced either selective LFPs or selective spiking responses and which passed the bootstrap test which 
Figure 6. Example of a microelectrode with selective LFP mean phase. (A) Averaged LFPs filtered in the $\theta$ band with the central frequency of the complex Morlet wavelet at $6 \mathrm{~Hz}$ (thick line) for three categories \pm 1 SEM (thin lines). (B) Normalized $p$ values retrieved from a test for a common mean direction. The black vertical line indicates the time point at which the mean phases were most different. The dash-dotted line corresponds to the significance level $p=.001$. (C) Phase distributions across trials taken at the moment of the largest difference among categories, as shown in (A) and (B) with the solid vertical line. Colored vectors show the mean phase direction. The length of the vector is proportional to the significance of the difference of the phase distribution for a given category from a uniform circular distribution (Rayleigh test). $p$ values are given next to the category names. Here, the phase distribution of the category "faces" significantly differs from a uniform circular distribution $\left(p<10^{-7}\right)$.
A

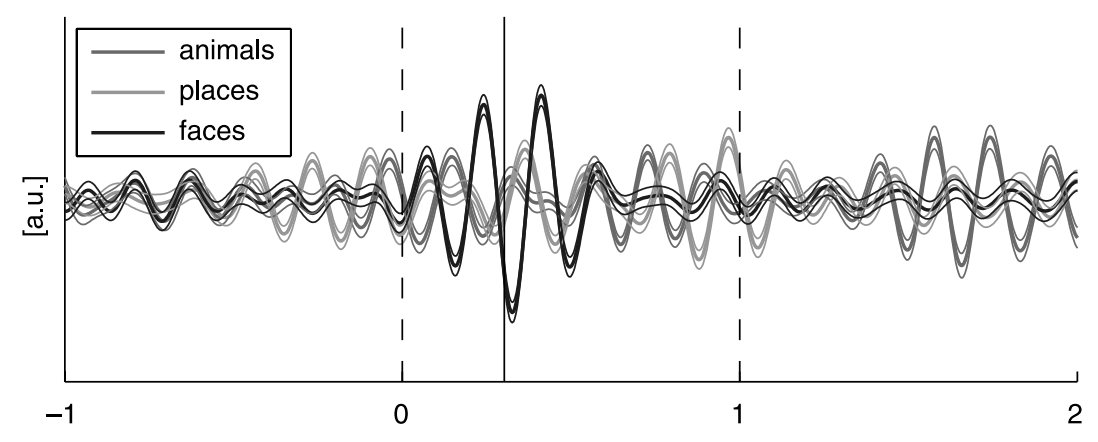

B

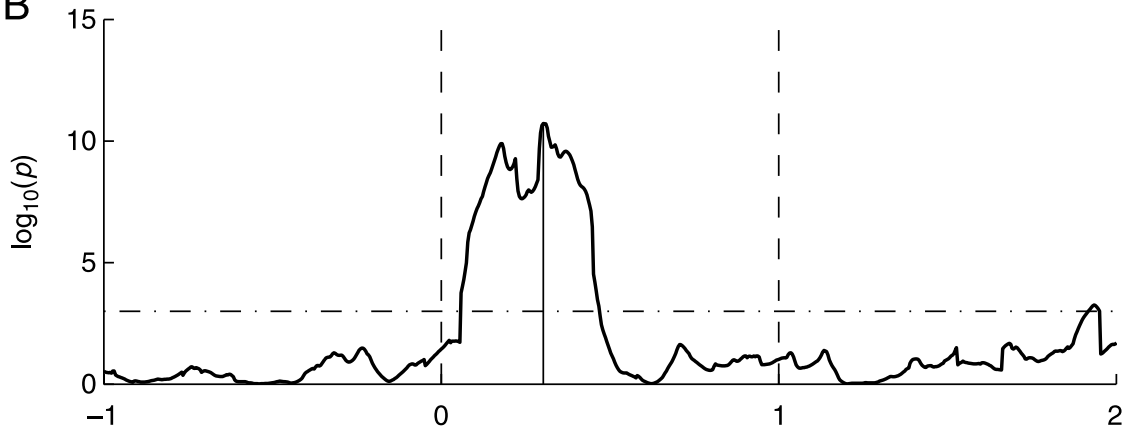

C

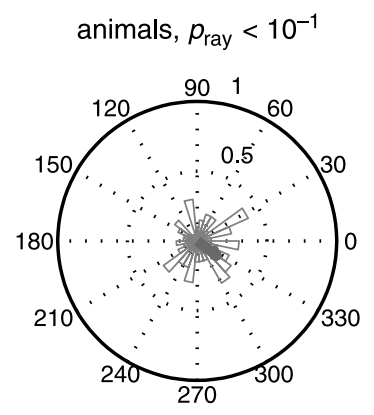

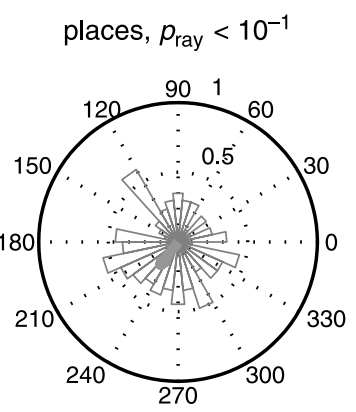

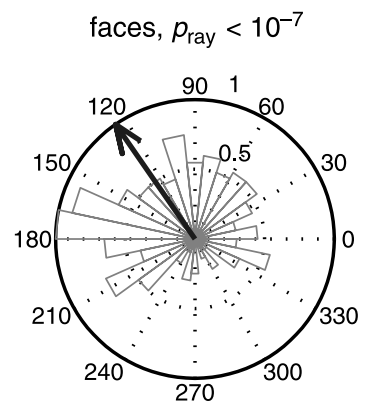

shuffles pictures between categories. However, only seven of them were selective for both the LFPs and the spiking activity. Six microelectrodes showed mean phase and spiking selectivity and 10 spike-selective microelectrodes showed power selectivity. The distribution of these channels across the different frequency bands is indicated with red bars in Figure 7.

\section{Decoding with LFPs and Spiking Activity}

We applied a linear decoding algorithm to the LFPs and the spiking activity recorded simultaneously from many microelectrodes in the MTL in order to ascertain how much information pertaining to the semantic category of the images can be inferred readout from the neural data. Here we use the term "decoding" in the computational sense, namely, we studied how reliably one can predict in each single trial the category identity of the stimulus given the firing of the neurons or the LFP activity. We studied the time profile of the decoding accuracy
(Methods) in the time interval $[-1: 2]$ sec. The inputs to the decoding algorithm were the number of spikes for each category or the mean value of the amplitude of the LFP defined in sliding windows of different sizes with $50 \%$ overlap. Only the activity of amplitude-selective microelectrodes (one-way ANOVA, $p<.01,15$ consecutive points) was taken as an input.

We found that the time profile of the decoding accuracy using spiking data increased with the length of the moving window (Figure 8B) and saturated for windows longer then 200 msec. For LFPs, the time profile remained approximately at the same level (Figure 8A) for different window sizes. Both for spiking and LFP data, and for all durations of the moving window, decoding accuracy during the baseline interval did not differ from chance $(t$ test, $p>.05)$. LFPs slightly outperformed the spiking activity only for very small window sizes of 10 and 20 msec. Moreover, for 10-msec windows, the classifier could barely distinguish between the categories using only spikes. This can be explained 
Figure 7. Number of microelectrodes with showing selectivity with LFP power and mean phase. The distribution of the (A) power and (B) mean phase-selective microelectrodes across different frequency bands of the LFPs. A total of 20 microelectrodes out of 475 showed category selectivity using the power analysis and 29 microelectrodes showed category selectivity using mean phase analysis. In principle, the same microelectrodes could be selective in different frequency bands. For the total number of selective microelectrodes, we count such electrodes only once. A large overlap is observed in selective microelectrodes according to LFP amplitude analysis and the mean phase analysis (15 microelectrodes).
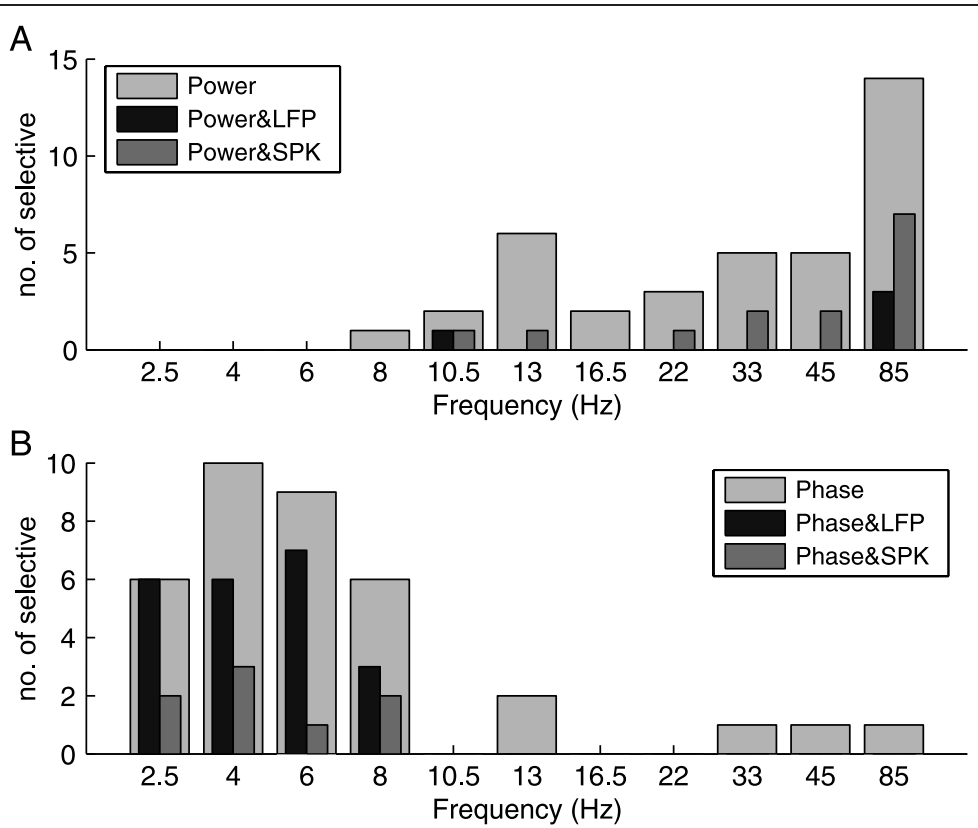

by the typically low firing rates of these neurons, considering that they may not produce a single spike during a 10-msec window. Combining the LFP and spiking activities increased the decoding accuracy for all window sizes between 10 and 200 msec (Figure 8C). The traces in Figure 8D show the decoding accuracies obtained with the LFPs, with the spikes and with both the LFPs and spikes for a window length of $20 \mathrm{msec}$. The red crosses indicate time points when decoding using the combination of the LFPs and spikes was significantly better (paired $t$ test, $p<.01$ ) than the decoding accuracy using only the amplitude of the LFPs, whereas the blue circles show the comparison between accuracy of the combined spikes and LFPs signal with the one using only spikes. For longer windows, decoding accuracy with combined LFPs and spikes input was also slightly better than accuracy obtained with each signal alone. It is notable that the time when the decoding accuracy became significantly different from chance was very similar for the LFPs and the spiking activity, around 300 msec after stimulus onset. The fact that we can infer category identity by applying a classifier to LFPs does not imply that the brain makes use of this information, but only that this information is present in the MTL and could be used by postsynaptic processes.

\section{DISCUSSION}

In previous studies, the spiking activity of single neurons in the human MTL was found to be selective to different categories of visual stimuli (Kreiman et al., 2000) and even invariant to different views of the same person or object (Quian Quiroga et al., 2005). The analysis of the LFPs recorded from the surface of the brain revealed faceselective LFPs in the extrastriate cortex (Allison, Ginter, et al., 1994; Allison, McCarthy, Nobre, Puce, \& Belger, 1994). Later studies using intracranial depth electrodes localized the source of the face-selective LFPs near the fusiform gyrus (Lachaux et al., 2005; Allison, Puce, Spencer, \& McCarthy, 1999), in good agreement with fMRI findings (Kanwisher, McDermott, \& Chun, 1997). In this article, we undertook a combined study of the LFPs and spiking activity recorded by the same microelectrodes to assess their object- and face-selectivity properties. We found that the spiking activity recorded from $56 \mathrm{mi}-$ croelectrodes was selective to semantic categories. The amplitude of the LFPs from a smaller number of microelectrodes (36) also showed category selectivity, but not necessarily to the category "faces."

We also found that the power in the $\gamma$ band was discriminative between categories but only in a small number of microelectrodes. This finding is similar to one reported by Oya, Kawasaki, Howard, and Adolphs (2002), who showed selectivity of the LFP $\gamma$ power in the amygdala in response to emotional faces. Although we did not find any power selectivity in lower frequency bands for a small number of microelectrodes, the mean phases of three categories were different in the $\delta(1-4 \mathrm{~Hz})$ and $\theta(4-8 \mathrm{~Hz})$ bands, but not in the $\gamma$ band (higher than $30 \mathrm{~Hz}$ following the definition in Engel, Fries, \& Singer, 2001). This suggests two possible mechanisms for selectivity of LFPs: via phase-locking in the lower frequency bands and/or via power increase at high frequencies. Because the power of $\delta$ and $\theta$ oscillations is significantly larger than the power of $\gamma$ oscillations (see the average LFP power spectra Figure S2), it is more efficient (i.e., less energy consuming 


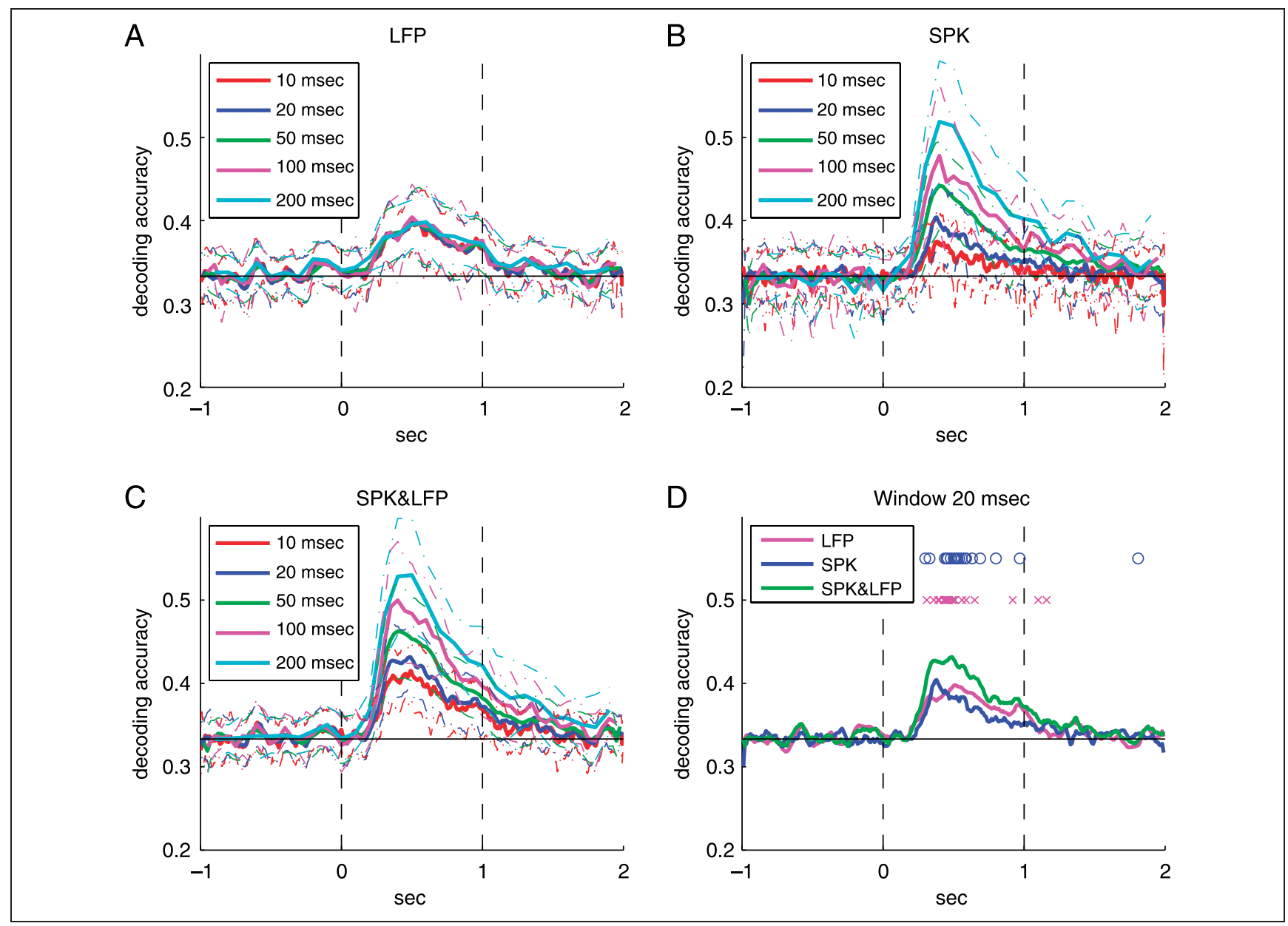

Figure 8. Time profile of decoding accuracy, using the spiking activity, the LFPs, and both signals together. Accuracy varies between perfect (1) and chance level (1/3). Decoding accuracy using (A) the average value of LFPs amplitude, (B) firing rates (SPK), and (C) their combination (SPK/LFP). The different curves on the (A,B,C) subplots correspond to window sizes of 10, 20, 50, 100, and 200 msec. Decoding accuracy with LFPs is nearly independent of the window size in this range, whereas accuracy with spikes (with or without LFP) increased with the window size. All curves were smoothed with a 5-point moving average. Dash-dotted lines show the confidence intervals obtained for the time points from a $t$ test comparison with chance level ( $1 / 3$, black line), $p<.01$. Decoding with LFPs, spikes, and their combination is shown in (D) for a window of size 20 msec. Optimal accuracy is achieved with the combination of LFPs and spiking activity. Crosses (circles) indicate time points where decoding for combination of the LFPs and spiking activity was significantly better than decoding using only LFPs (only spikes) (paired $t$ test, $p<.01$ ).

and faster) to transmit information by modulation of $\gamma$ power. On the other hand, $\gamma$ oscillations are much faster than $\delta$ or $\theta$ ones, therefore, a small jitter in $\gamma$ oscillations will destroy their synchronization but would hardly influence slow $\delta$ and $\theta$ oscillations. Previous studies with rats performing spatial tasks also found a phase locking of spikes with the ongoing activity in the $\theta$ band (Siapas, Lubenov, \& Wilson, 2005). Synchronization of $\gamma$ oscillations was suggested as possible mechanism for information processing (Singer, 1999). The vast majority of the LFP amplitude-selective microelectrodes were found in the hippocampus (30 out of 36). The relative number of hippocampal-selective microelectrodes (16\%) was three times larger than in all other areas (Figure 4). At the same time, the relative number of spike-selective electrodes was evenly distributed across all four investigated areas. This discrepancy suggests that the category-selective LFPs are either the result of local processing within the hippocam- pus or that the hippocampus receives a category-specific input from adjacent areas.

Only a small overlap was observed between spikes and LFP selective microelectrodes. This lack of correlation supports the view that neurons in the MTL are only weakly spatially clustered in terms of the semantic categories (faces, places, animals). This result is in line with the weak correlation between the object-selective LFPs and object-selective spiking activity recently reported in monkey IT (Kreiman et al., 2006). It also suggests that spiking activity and LFPs contain different information about stimulus category.

Halgren et al. (1980) recorded LFPs in the human MTL during an "oddball" paradigm and found a P300 evoked potential well known from surface EEG measurements. Some studies argue for a generation of the P300 in the hippocampal formation and amygdala (Halgren, Marinkovic, \& Chauvel, 1998; McCarthy, Wood, Williamson, \& Spencer, 


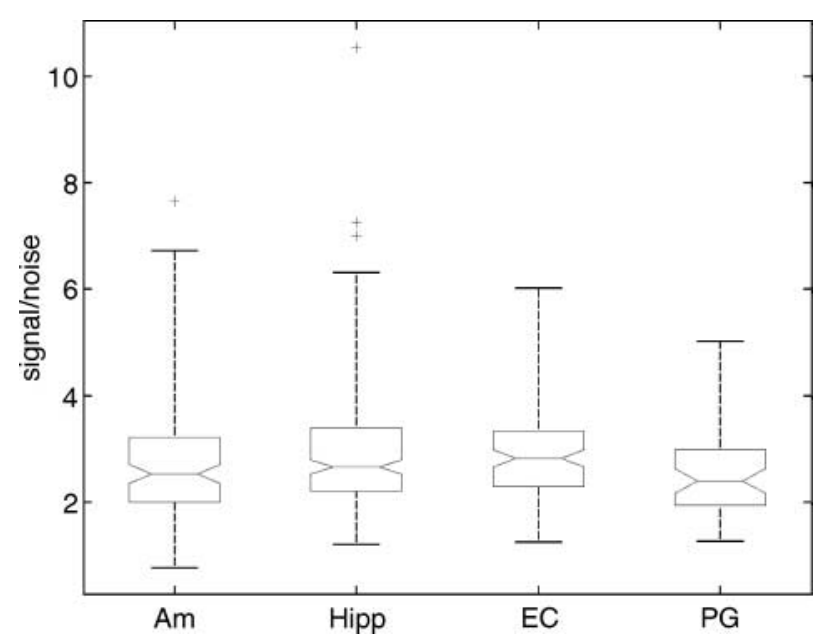

Figure S1. Signal-to-noise ration indifferent brain areas. Boxplot of the signal-to-noise ratios in different brain areas. Horizontal middle lines represent medians of distributions of signal-to-noise ratios.

1989). To check whether the evoked potentials which we observed were task-dependent, we repeated our experiment in one patient without a task (i.e., the patient was asked to passively look at the pictures presented on the screen for $500 \mathrm{msec}$ ). We found two (out of 24 microelectrodes analyzed for this session) LFP amplitudeselective microelectrodes (Figure S4). This corresponds to the $8 \%$ of LFP-selective microelectrodes found with task and argues in favor of task independence.

The LFPs represent the average dendrosomatic activity of presynaptic signals of large neuronal populations
(Logothetis, 2003; Mitzdorf, 1985). Therefore, the observed selectivity of LFPs in the MTL might be caused by specific pattern of dendritic activity arising, for example, from the prefrontal cortex, which has been reported to be involved in categorical representation of visual stimuli (Freedman, Riesenhuber, Poggio, \& Miller, 2001) or by local synaptic circuitry, which is differentially activated for different categories of visual stimuli. It is not possible to distinguish among these possibilities with the current data.

The weak correlation in the selectivity properties of LFPs and spikes suggests that they reflect two different aspects of brain activity. A similar disassociation between LFPs and spikes was also observed in macaque V1 (Henrie \& Shapley, 2005). These authors hypothesized that the network activity captured by LFPs originates from inhibitory interneurons, whereas single-unit activity is largely biased toward pyramidal neurons.

This relative independence observed in the selectivity of the spiking and LFPs activities is compatible with our decoding analysis. Reading out both the mean number of spikes as well as the mean amplitude of the LFP allowed us to infer the identity of the category of the visual stimulus significantly better than using either measure by itself. This effect was more pronounced when information from short time windows was used for classification.

\section{SUPPLEMENTARY MATERIAL}

\section{Signal-to-noise Ratio in Different Brain Areas}

One possible explanation for the fact that 30 out of 36 LFP-selective microelectrodes were found in the hippo-
Figure S2. Average LFPS power spectrum. Magenta (blue) solid line shows a power spectrum averaged across stimulus presentation [0:1] sec (baseline [-1:0] sec) interval for all analyzed LFPs. Dash-dotted lines depict least-squares logarithmic fit, $f^{-\alpha}$. For stimulus presentation interval average $\alpha$ was found to be equal to $2.01 \pm-0.02$, (mean \pm SEM) and for the baseline interval to be $2.04 \pm 0.02$, (mean \pm SEM). Note that the magenta and blue curves practically almost overlap. Drop at $60 \mathrm{~Hz}$ is due to the digital notch filter applied at $60 \mathrm{~Hz}$.

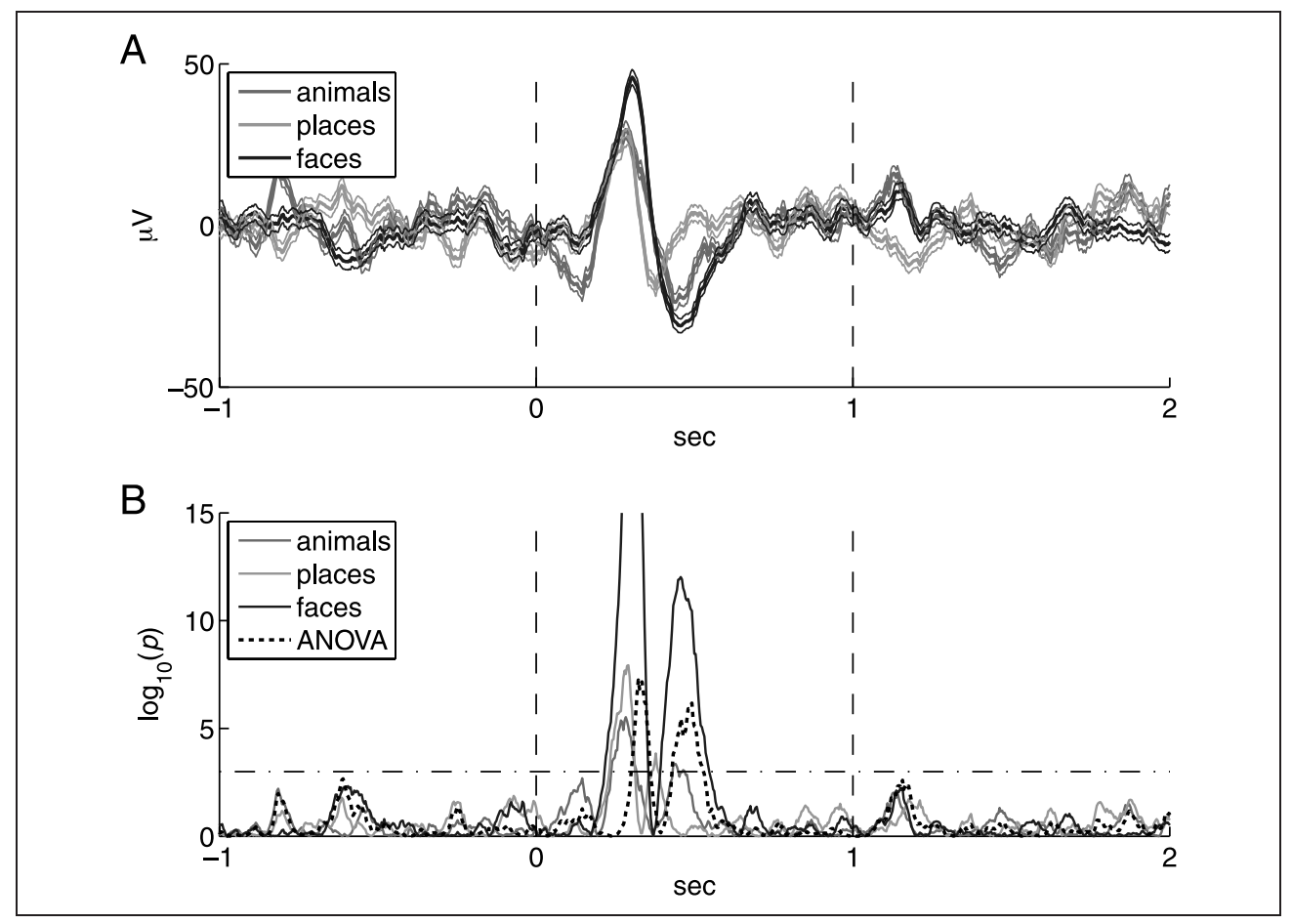


Figure S3. Latency of selectivity versus latency of responsiveness. (A) Averaged LFPs (thick line) for the three categories \pm 1 SEM (thin lines) corresponding to an electrode implanted in the right amygdala. (B) Normalized $p$ values obtained with the ANOVA test of selectivity (black thick line) and the $t$ tests or responsiveness for each category (color lines). A sample-by-sample $t$ test was applied to the distribution of LFPs values for each category and each time point during stimulus presentation with the distribution of all LFPs values during the baseline interval. The dash-dotted line corresponds to a significance of .001. The ANOVA trace first crosses the significance level of $p=.001$ at $\sim 300 \mathrm{msec}$, whereas the $t$ test reached significance earlier at $\sim 230$ msec.

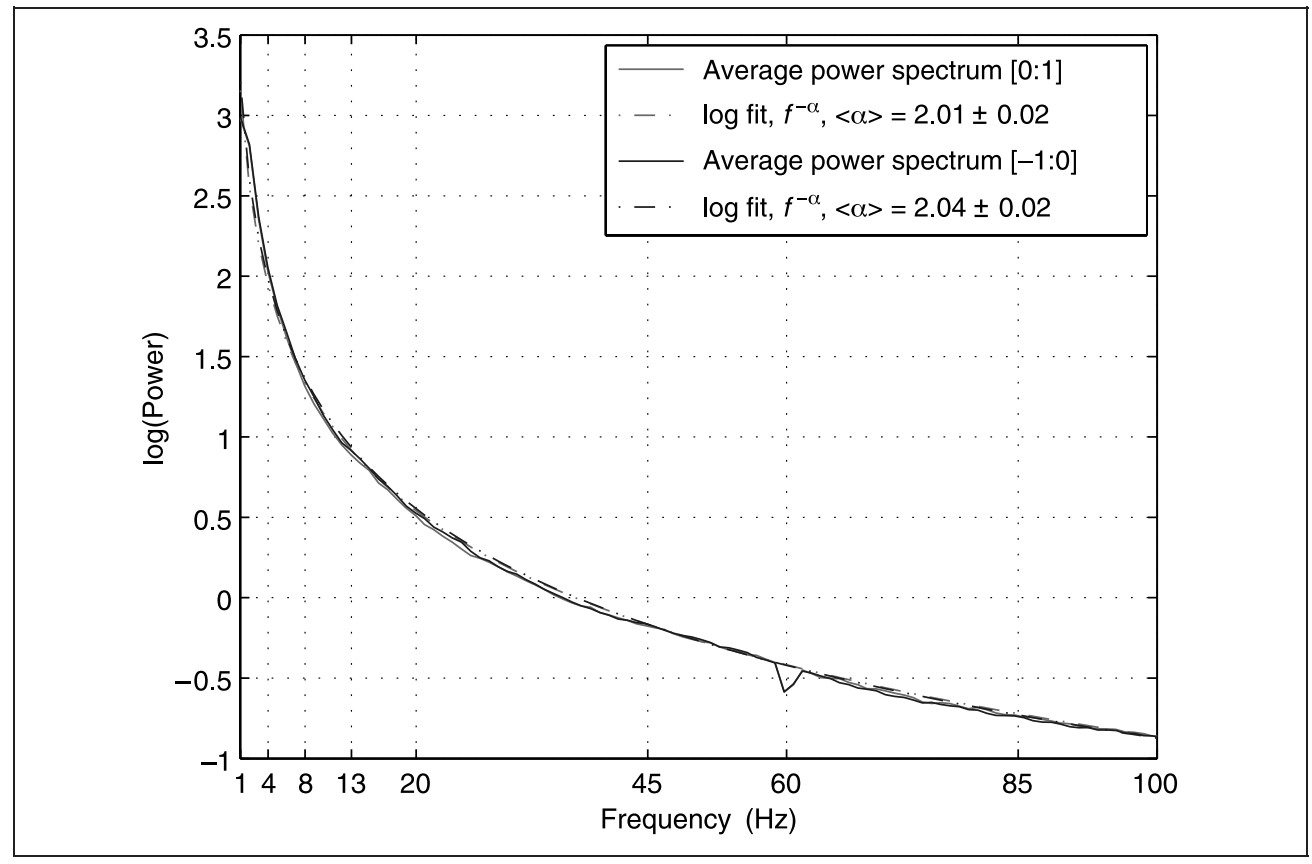

campus could be that given its anatomical structure, the signal-to-noise ratio in the hippocampus is better than in the amygdala. We statistically compared signal-to-noise ratios for evoked potentials in different brain areas (see Figure S1). Signal-to-noise ratio was estimated for each microelectrode as the ratio of the maximum amplitude of the evoked potential to the standard error of the mean. Mean values of signal-to-noise ratios in the hippocampus and entorhinal cortices were found to be higher than in the amygdala and parahippocampal gyrus. But
Figure S4. Time-resolved selectivity analysis. Selectivity analysis of LFPs recorded during a passive viewing task. Each stimulus was presented for 500 msec. (A) Averaged LFPs (thick line) for the three categories \pm 1 SEM (thin lines). (B) Normalized $p$ values obtained with the ANOVA test. The dash-dotted line corresponds to a significance of $p=.001$. All three categories show significant response but there is a clear difference between the category "faces" and two other categories.

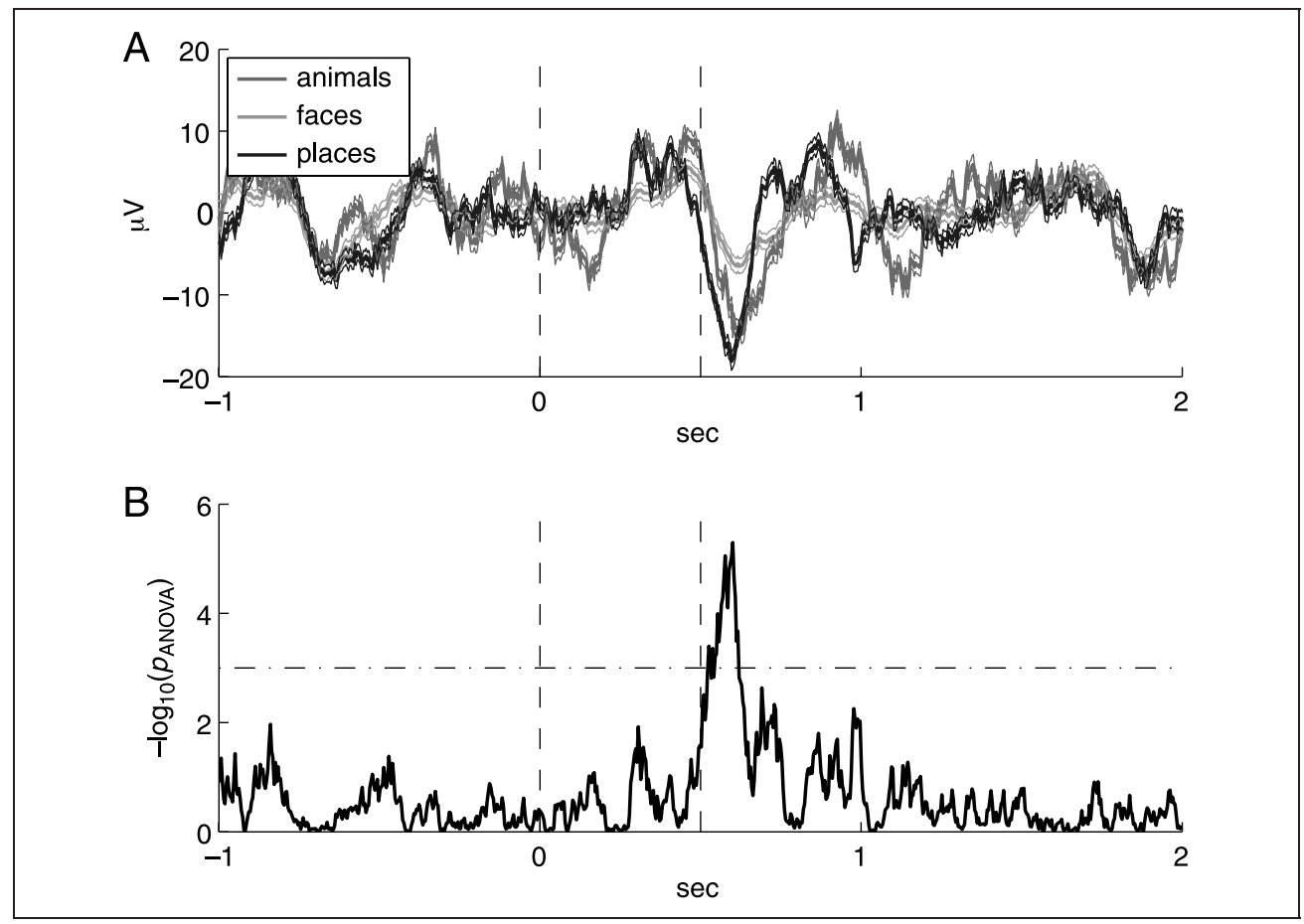


the difference was not statistically significant ( $t$ test, $p>$ .05 for hippocampus vs. amygdala, and hippocampus vs. entorhinal cortex), and was only slightly significant for the hippocampus versus parahippocampal gyrus $(.01<$ $p<.05)$. Therefore, the difference between signal-tonoise ratios in different brain areas cannot explain the larger selectivity of LFPs found in the hippocampus than in any other area.

\section{Numbers of Responsive LFPs and Units to Different Categories}

For the spiking activity a $t$ test comparison between the firing rate during the poststimulus [300:1000] msec and baseline [1000:-300] msec intervals was used to define the responsive category. For LFPs we applied a sampleby-sample $t$ test comparing the distribution of LFP values for each category and each time point during stimulus presentation, with the distribution of all LFP values during the baseline interval. The LFPs were defined to be responsive to a category if the $t$ test trace crossed a significance level of $p=.001$ for at least 15 consecutive points (see Figure S3). The following tables provide information about the relative number of responsive units and LFPs across different categories as well as different brain areas.

\begin{tabular}{lccc}
\hline & Animals & Faces & Places \\
\hline LFP & $24(67 \%)$ & $35(97 \%)$ & $14(39 \%)$ \\
All & 3 & 2 & 1 \\
AM & 21 & 30 & 13 \\
Hipp & 0 & 3 & 0 \\
EC & 0 & 0 & 0 \\
PG & & & \\
Spikes & $32(48 \%)$ & $31(47 \%)$ & $26(39 \%)$ \\
All & 17 & 15 & 12 \\
AM & 10 & 7 & 8 \\
Hipp & 1 & 7 & 1 \\
EC & 4 & 2 & 5 \\
PG & & & \\
\hline
\end{tabular}

Note that the same unit might show a significant increase in firing rate for one category and a significant decrease for another one (see Figure 2). For the LFP, we use a time-resolved measure, therefore, the LFP from the same microelectrode can respond to several categories but can be different at different time points. For example, the LFPs in Figure S3 are responsive for all three image categories. Yet at the same time, an ANOVA test shows that the LFP differs among the three image categories at somewhat different times. Thus, in this example, the LFP can discriminate among the three categories but at different times.

\section{Acknowledgments}

We thank all patients for their participation and Eric Behnke, Tony Fields, Emily Ho, Eve Isham, and Charles Wilson for technical assistance. We also would like to thank Gabriel Kreiman and Daniel Rizzuto for helpful discussions. This work was supported by grants from the NINDS, NIMH, NSF, DARPA, EPSRC, the Office of Naval Research, the W.M. Keck Foundation Fund for Discovery in Basic Medical Research, the Gordon Moore Foundation, the Sloan Foundation, and the Swartz Foundation for Computational Neuroscience.

Reprint requests should be sent to Dr. Alexander Kraskov, Sobell Department of Motor Neuroscience, UCL Institute of Neurology, Queen Square, London WC1N 3BG, UK, or via e-mail: a.kraskov@ion.ucl.ac.uk.

\section{REFERENCES}

Allison, T., Ginter, H., McCarthy, G., Nobre, A. C., Puce, A., Luby, M., et al. (1994). Face recognition in human extrastriate cortex. Journal of Neurophysiology, 71, 821-825.

Allison, T. T., McCarthy, G. G., Nobre, A. A., Puce, A. A., \& Belger, A. A. (1994). Human extrastriate visual cortex and the perception of faces, words, numbers, and colors. Cerebral Cortex, 4, 544.

Allison, T. T., Puce, A. A., Spencer, D. D. D., \& McCarthy, G. G. (1999). Electrophysiological studies of human face perception: I. Potentials generated in occipitotemporal cortex by face and non-face stimuli. Cerebral Cortex, 9, 415.

Bechtereva, N. P., \& Abdullaev, Y. G. (2000). Depth electrodes in clinical neurophysiology: Neuronal activity and human cognitive function. International Journal of Psychophysiology, 37, 11-29.

Blair, R. C., \& Karniski, W. (1993). An alternative method for significance testing of wave-form difference potentials. Psychophysiology, 30, 518-524.

Buchwald, J. S. (1965). Comparison of multiple-unit and electroencephalogram activity recorded from same brain sites during behavioural conditioning. Nature, 205, 1012.

Duda, O. H., Hart, P. E., \& Stork, D. G. (2001). Pattern classification (2nd ed.). New York: Wiley.

Engel, A. K., Fries, P., \& Singer, W. (2001). Dynamic predictions: Oscillations and synchrony in top-down processing. Nature Reviews Neuroscience, 2, 704-716.

Engel, A. K., Moll, C. K. E., Fried, I., \& Ojemann, G. A. (2005). Invasive recordings from the human brain: Clinical insights and beyond. Nature Reviews Neuroscience, 6, $35-47$.

Fisher, N. I. (1995). Statistical analysis of circular data. Cambridge: Cambridge University Press.

Freedman, D. J., Riesenhuber, M., Poggio, T., \& Miller, E. K. (2001). Categorical representation of visual stimuli in the primate prefrontal cortex. Science, 291, 312-316.

Fried, I., MacDonald, K. A., \& Wilson, C. L. (1997). Single neuron activity in human hippocampus and amygdala during recognition of faces and objects. Neuron, 18, 753-765.

Fried, I., Wilson, C. L., Maidment, N. T., Engel, J., Behnke, E., Fields, T. A., et al. (1999). Cerebral microdialysis combined 
with single-neuron and electroencephalographic recording in neurosurgical patients [Technical note]. Journal of Neurosurgery, 91, 697-705.

Fromm, G. H. (1967). Relationship between neuron activity and cortical steady potentials. Electroencephalography and Clinical Neurophysiology, 22, 159.

Grossmann, A., Kronland-Martinet, R., \& Morlet, J. (1989). Reading and understanding continuous wavelet transforms. In J. Combes, A. Grossmann, \& P. Tchamitchian (Eds.), Wavelets: Time-frequency methods and phase space. Berlin: Springer.

Guthrie, D., \& Buchwald, J. S. (1991). Significance testing of difference potentials. Psychophysiology, 28, 240-244.

Haberly, L. B., \& Shepherd, G. M. (1973). Current-density analysis of summed evoked potentials in opossum prepyriform cortex. Journal of Neurophysiology, 36, 789-802

Halgren, E., Marinkovic, K., \& Chauvel, P. (1998). Generators of the late cognitive potentials in auditory and visual oddball tasks. Electroencephalography and Clinical Neurophysiology, 106, 156-164.

Halgren, E., Squires, N. K., Wilson, C. L., Rohrbaugh, J. W., Babb, T. L., \& Crandall, P. H. (1980). Endogenous potentials generated in the human hippocampal-formation and amygdala by infrequent events. Science, 210, 803-805.

Henrie, J. A., \& Shapley, R. (2005). LFP power spectra in V1 cortex: The graded effect of stimulus contrast. Journal of Neurophysiology, 94, 479-490.

John, E. R. (1967). Effects of visual form on evoked response. Science, 155, 1439.

John, E. R. (1972). Switchboard versus statistical theories of learning and memory. Science, 177, 850.

Kanwisher, N., McDermott, J., \& Chun, M. M. (1997). The fusiform face area: A module in human extrastriate cortex specialized for face perception. Journal of Neuroscience, 17, 4302-4311.

Kreiman, G., Hung, C. P., Kraskov, A., Quian Quiroga, R., Poggio, T., \& DiCarlo, J. (2006). Object selectivity of local field potentials and spikes in the macaque inferior temporal cortex. Neuron, 49, 433-445.

Kreiman, G., Koch, C., \& Fried, I. (2000). Category-specific visual responses of single neurons in the human medial temporal lobe. Nature Neuroscience, 3, 946-953.

Lachaux, J. P., George, N., Tallon-Baudry, C., Martinerie, J., Hugueville, L., Minotti, L., et al. (2005). The many faces of the gamma band response to complex visual stimuli. Neuroimage, 25, 491-501.

Lachaux, J. P., Rudrauf, D., \& Kahane, P. (2003). Intracranial EEG and human brain mapping. Journal of PhysiologyParis, 97, 613-628.

Liu, J., \& Newsome, W. T. (2006). Local field potential in cortical area MT: Stimulus tuning and behavioral correlations. Journal of Neuroscience, 26, 7779-7790.
Logothetis, N. K. (2003). The underpinnings of the BOLD functional magnetic resonance imaging signal. Journal of Neuroscience, 23, 3963-3971.

McCarthy, G., Wood, C. C., Williamson, P. D., \& Spencer, D. D. (1989). Task-dependent field potentials in human hippocampal-formation. Journal of Neuroscience, 9, 4253-4268.

Mehring, C., Rickert, J., Vaadia, E., de Oliveira, S. C., Aertsen, A., \& Rotter, S. (2003). Inference of hand movements from local field potentials in monkey motor cortex. Nature Neuroscience, 6, 1253-1254.

Mitzdorf, U. (1985). Current source-density method and application in cat cerebral cortex: Investigation of evoked potentials and EEG phenomena. Physiological Reviews, 65, 37-100.

Oya, H., Kawasaki, H., Howard, M. A., \& Adolphs, R. (2002). Electrophysiological responses in the human amygdala discriminate emotion categories of complex visual stimuli. Journal of Neuroscience, 22, 9502-9512.

Pesaran, B., Pezaris, J. S., Sahani, M., Mitra, P. P., \& Andersen, R. A. (2002). Temporal structure in neuronal activity during working memory in macaque parietal cortex. Nature Neuroscience, 5, 805-811.

Quian Quiroga, R., Nadasdy, Z., \& Ben-Shaul, Y. (2004). Unsupervised spike detection and sorting with wavelets and superparamagnetic clustering. Neural Computation, 16, 1661-1687.

Quian Quiroga, R., Reddy, L., Kreiman, G., Koch, C., \& Fried, I. (2005). Invariant visual representation by single neurons in the human brain. Nature, 435, 1102-1107.

Rizzuto, D. S., Madsen, J. R., Bromfield, E. B., Schulze-Bonhage, A., Seelig, D., Aschenbrenner-Scheibe, R., et al. (2003). Reset of human neocortical oscillations during a working memory task. Proceedings of the National Academy of Sciences, U.S.A., 100, 7931-7936.

Scherberger, H., Jarvis, M. R., \& Andersen, R. A. (2005). Cortical local field potential encodes movement intentions in the posterior parietal cortex. Neuron, 46, 347-354.

Siapas, A. G., Lubenov, E. V., \& Wilson, M. A. (2005). Prefrontal phase locking to hippocampal theta oscillations. Neuron, 46, 141-151.

Singer, W. (1999). Neuronal synchrony: A versatile code for the definition of relations? Neuron, 24, 49-65.

TallonBaudry, C., Bertrand, O., Delpuech, C., \& Pernier, J. (1997). Oscillatory gamma-band (30-70 Hz) activity induced by a visual search task in humans. Journal of Neuroscience, 17, 722-734.

Verzeano, M., Crandall, P. H., \& Dymond, A. (1971). Neuronal activity of amygdala in patients with psychomotor epilepsy. Neuropsychologia, 9, 331-344.

Wyler, A. R., Ojemann, G. A., \& Ward, A. A. (1982). Neurons in human epileptic cortex-Correlation between unit and EEG activity. Annals of Neurology, 11, 301-308. 